Research Article

\title{
DBU catalysis in micellar medium: an environmentally benign synthetic approach for triheterocyclic $4 \mathrm{H}$-pyrimido[2,1-b] benzothiazole derivatives
}

\author{
Shiwani Singh ${ }^{1} \cdot$ Jaggi Lal ${ }^{1}$ (i)
}

Received: 30 March 2020 / Accepted: 9 June 2020 / Published online: 23 June 2020

(c) Springer Nature Switzerland AG 2020

\begin{abstract}
An efficient, simple, practical and scalable approach for the preparation of pharmaceutically important scaffold $4 H$-pyrimido[2,1-b]benzothiazoles using one-flask three-component cyclo-condensation of $\beta$-ketoester, substituted aromatic aldehydes and 2-aminobenzothiazole under micellar medium as an environmentally benign protocol is reported. The reaction is catalyzed by DBU and CPB as a green, easily available, cheap and sustainable catalyst. The product formation takes shorter duration with good to excellent yield of the products. The method is also demonstrated on multi-gram level. The Atom economy, environmentally benign, easy to work-up, no use of hazardous solvents, short reaction time are the most outstanding advantages of the developed methodology.
\end{abstract}

\section{Graphic abstract}
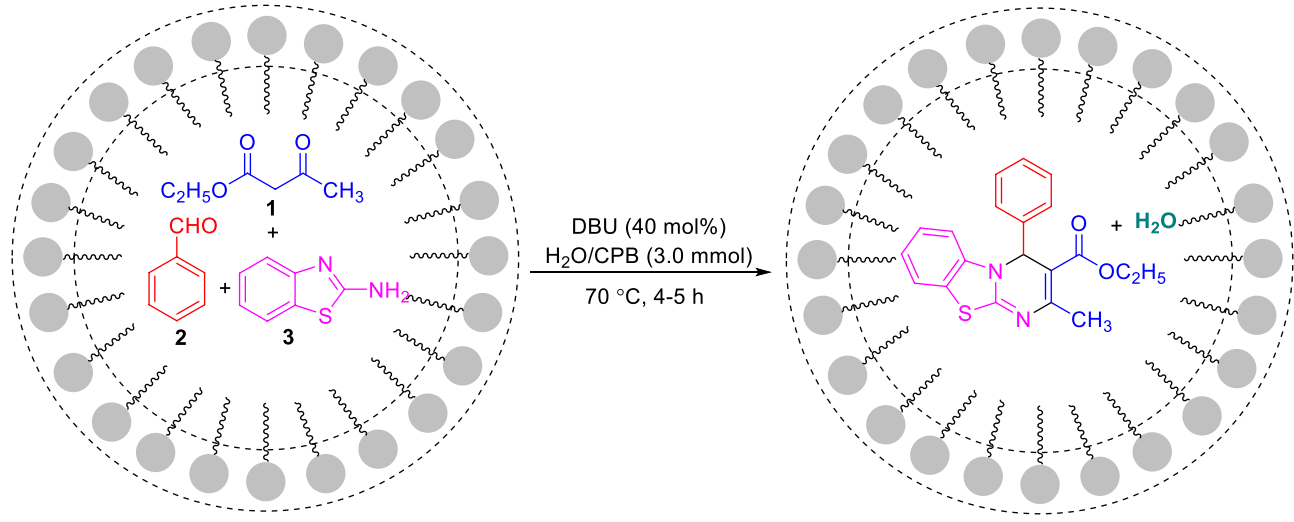

Keywords DBU · Micellar medium · Green Synthesis · Scalable Synthesis · Atomic Economy

\section{Introduction}

In heterocyclic chemistry the development of novel synthetic methodologies to facilitate the preparation of biologically active molecules is an important area of research.
In this regard, efforts have been constantly made to introduce new more efficient and environment compatible methodologies. One of the most attractive approaches to address this challenge is a search for commonly employed organic solvents from various health and environmental

\footnotetext{
Jaggi Lal, jaggitajagra@gmail.com | 'Department of Chemistry, Dr. K.N. Modi University, Newai 304021, Rajasthan, India.
} 
issues [1]. Keeping environmental concerns in mind, water would be the most attractive solvent to carry out chemical reactions, since it is cheap, safe, non-toxic and poses no threat to the environment [2]. As far as green chemistry is concerned, water may be used to carry out an organic transformation [3]. However, most of the organic compounds are not soluble in water, which is more important factor to carry out an organic transformation, in this case surface active agent (surfactant) may be added for the improvement of the solubility via the formation of micelles [4].

One-pot multicomponent reactions have gained a considerable attention for organic chemists and medicinal chemists [5-7] since they offer significant advantages over conventional stepwise synthetic procedures for promoting new reactions and for the development of straightforward synthetic routes to bioactive heterocycles [8]. Due to the wide spread applications of fused ring compounds (Fig. 1) as fluorescent dye (a) [9], photosensitizer (b) $[10,11]$ and pharmaceutical (c, d, e, f) [12].

Triheterocyclic $4 H$-pyrimido $[2,1-b]$ benzothiazoles are the most important class of heterocyclic molecules and their synthesis has received much attention in the field of medicinal chemistry due to their wide range of biological activities [13] such as anticonvulsant [14], antiviral [15], fungicidal [16], antiallergic [17] antimicrobial [18] anti-mycobaterial [19], anti-inflammatory [20], antiproliferative [21] antiviral [22] and antitumor [23] activities. Among the 2-aminobenzothiazole based compounds, $4 \mathrm{H}$-pyrimido[2,1-b]benzothiazoles are of interest and have great potential for further synthetic transformations. In literature, various methods for the synthesis of $4 H$-pyrimido[2,1-b] benzothiazoles via multicomponent synthesis such as $\mathrm{Fe}_{3} \mathrm{O}_{4} @$ @nano-cellulose/Cu(II) [24], solvent-free [25] tetrabutylammonium hydrogen sulfate
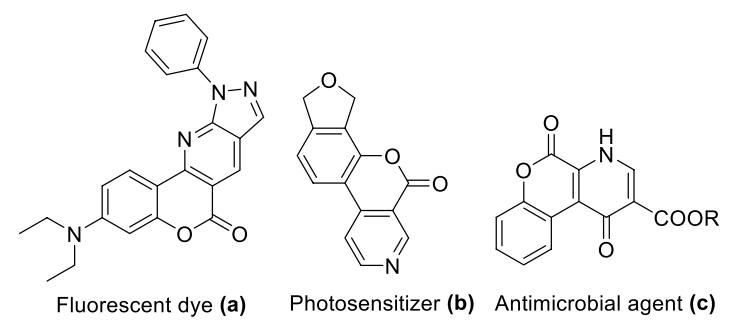

Photosensitizer (b) Antimicrobial agent (c)
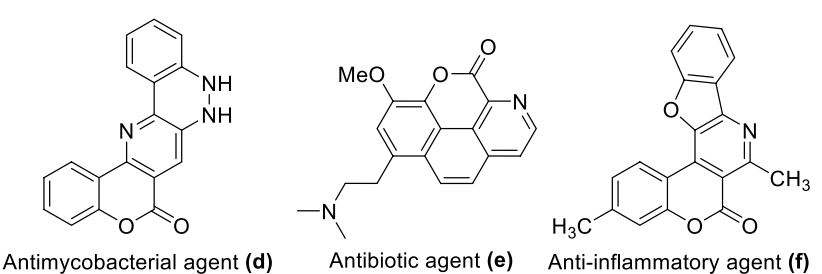

Antimycobacterial agent (d) Antibiotic agent (e)

Anti-inflammatory agent (f)

Fig. 1 Structures of some important fused heterocyclic compounds

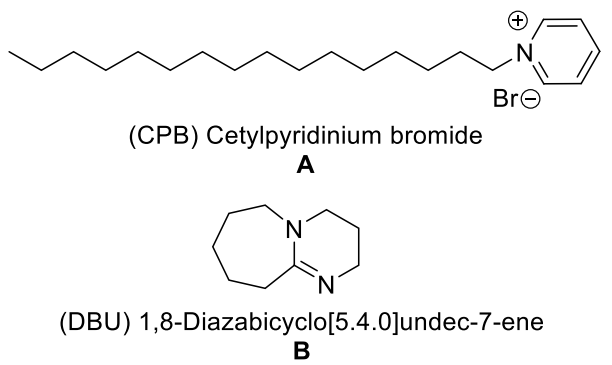

Fig. 2 Structures of commercially available surfactant (a) and catalyst (b)

(TBAHS) [26], iron fluoride [27], 1,1,3,3-N, $N, N, N^{\prime} N^{\prime}$-tetramethylguanidinium trifluoroacetate (TMGT) [28], $\mathrm{N}$-sulfonic acid modified poly(styrene-maleic anhydride) $\left(\mathrm{SMI}^{-} \mathrm{SO}_{3} \mathrm{H}\right)$ [29], $\mathrm{Fe}_{3} \mathrm{O}_{4} @$ nano-cellulose/ $\mathrm{TiCl}$ [30] anhydrous zinc chloride [31], $N, N^{\prime}$-dichlorobis(2,4,6-trichlorophenyl)urea [32] and boric acid [33] have been employed for their synthesis. However, in modern era green methodologies are desirable, because many of the reported methods are unsatisfactory as they involve the use of hazardous solvents, catalyst loadings up to $30 \mathrm{~mol} \%$, low yields of the products, drastic reaction conditions, long reaction durations and tedious isolation procedures have been described. These drawbacks of reported procedures make further improvements for the construction of $4 H$-pyrimido $[2,1-b]$ benzothiazoles essential.

According to literature, DBU (1,8-Diazabicyclo(5.4.0) undec-7-ene) has been found to catalyzed many organic reactions [34]. In addition to the DBU, surfactants also play an important role in catalysis chemistry [35] the structures of DBU and CPB are given in the Fig. 2.

Continuing our interest on the development of new methodologies for the synthesis of biologically active compounds [36-41] herein we wish to develop new protocol for the synthesis of biologically important triheterocyclic 4H-Pyrimido[2,1-b] benzothiazoles using DBU (1,8-Diazabicyclo(5.4.0)undec-7-ene) as an organic base catalyst in the presence of CPB (Cetylpyridinium bromide) surfactant in micellar media.

\section{Experimental}

\subsection{General remarks}

All the synthesized compounds have been identified by elemental analysis and by comparing their melting points with the authentic samples. Melting points were recorded on an electrical melting point apparatus and are reported uncorrected. The IR spectra were recorded on a Shimadzu IR spectrophotometer on KBR pellate, ${ }^{1} \mathrm{H}$ and ${ }^{13} \mathrm{C}$ NMR 
spectra were recorded on Bruker Avance II on $400 \mathrm{MHz}$ and $100 \mathrm{MHz}$ in $\mathrm{CDCl}_{3}$ solvent, mass spectra were recorded on mass spectrometer. TLC of the compounds was checked on silica gel G (E. Merck, India) plates. DBU and other reagents used in the experiments were purchased from Sigma Aldrich, Rankem and Spectrochem chemical companies.

\subsection{Synthesis of triheterocyclic $4 H$-pyrimido $[2,1-b]$ benzothiazole derivatives (4a-p)}

A mixture of ethyl acetoacetate $1(1.0 \mathrm{mmol})$, substituted aromatic aldehydes $\mathbf{2}(1.0 \mathrm{mmol}), 2$-aminobenzothiazole $3(1.0 \mathrm{mmol})$ and DBU $(60 \mathrm{mg}, 0.4 \mathrm{mmol})$ was taken in a $2 \mathrm{ml}$ homogeneous aqueous solution of CPB $(3.0 \mathrm{mmol}$, $1.092 \mathrm{~g}$ ) in a $10 \mathrm{ml}$ oven dried reaction vial. Heat the content at about $60^{\circ} \mathrm{C}$ for $3-4 \mathrm{~h}$. The progress of the reaction was monitored by TLC in ethyl acetate-hexane $(8: 2 \mathrm{v} / \mathrm{v})$. After completion of the reaction, $5 \mathrm{ml}$ water was added to the content. The solid product was collected by filtration over a thin Celite pad using vacuum pump and further purified by recrystallization from hot ethanol.

\subsection{Gram scaled-up synthesis of Ethyl-2-me- thyl-4-(phenyl)-4H-pyrimido[2,1-b] [1, 3] benzothia-zole-3-carboxylate (4a)}

A mixture of ethyl acetoacetate $1(5.0 \mathrm{mmol})$, benzaldehyde $2(5.0 \mathrm{mmol}), 2$-aminobenzothiazole $3(5.0 \mathrm{mmol})$ and DBU (300 mg, $0.4 \mathrm{mmol}$ ) was taken in a $20 \mathrm{ml}$ homogeneous aqueous solution of CPB $(15.0 \mathrm{mmol}, 5.46 \mathrm{~g})$ in a $50 \mathrm{ml}$ oven dried reaction vial. Heat the content at about $60^{\circ} \mathrm{C}$ for $4 \mathrm{~h}$. The progress of the reaction was monitored by TLC in ethyl acetate-hexane (8:2 v/v). After completion of the reaction, $5 \mathrm{ml}$ water was added to the content. The solid product was collected by filtration over a thin Celite pad using vacuum pump and further purified by recrystallization from hot ethanol.

\subsection{Characterization data of some selected synthe- sized compounds}

\subsubsection{Ethyl-2-methyl-4-(phenyl)-4H-pyrimido[2,1-b] [1, 3]} benzothiazole-3-carboxylate (4a)

Pale yellow crystals, IR $\left(\lambda \max \mathrm{cm}^{-1}\right): 3041,2965,1672$, $1583,1468,1241,743 ;{ }^{1} \mathrm{H}$ NMR $\left(400 \mathrm{MHz}, \mathrm{CDCl}_{3}\right): \delta 1.27$ $\left(3 \mathrm{H}, \mathrm{t}, J=14.21 \mathrm{~Hz}, \mathrm{CH}_{2} \mathrm{CH}_{3}\right), 2.44\left(3 \mathrm{H}, \mathrm{s}, \mathrm{CH}_{3}\right), 4.21(2 \mathrm{H}, \mathrm{m}$, $\left.\mathrm{CH}_{2} \mathrm{CH}_{3}\right), 6.39(1 \mathrm{H}, \mathrm{s},-\mathrm{CH}), 7.08-7.48(9 \mathrm{H}, \mathrm{m}, \mathrm{ArH}) ;{ }^{13} \mathrm{C} \mathrm{NMR}$ $\left(400 \mathrm{MHz}, \mathrm{CDCl}_{3}\right): 165.41,162.61,154.11,141.21,137.41$, 128.22, 126.76, 122.28, 111.61, 103.53, 58.36, 56.89, 24.22, 14.84; ESI-MS: $\mathrm{m} / \mathrm{z}$ Calcd for $\mathrm{C}_{20} \mathrm{H}_{18} \mathrm{~N}_{2} \mathrm{O}_{2} \mathrm{~S} 350.44$, found $[\mathrm{M}+\mathrm{H}]^{+}$351.2.
2.4.2 Ethyl-2-methyl-4-(4-nitro phenyl)-4H-pyrimido[2,1-b] $[1,3]$ benzothiazole-3-carboxylate (4b)

Yellow powder, IR $\left(\lambda \operatorname{max~cm} \mathrm{cm}^{-1}\right): 3351,2942,1621,1517$, $1271,1118,961-814{ }^{1}{ }^{1} \mathrm{H} \mathrm{NMR}\left(400 \mathrm{MHz}, \mathrm{CDCl}_{3}\right): \delta 1.36(3 \mathrm{H}$, $\left.\mathrm{s}, \mathrm{CH}_{2} \mathrm{CH}_{3}\right), 2.51\left(3 \mathrm{H}, \mathrm{s}, \mathrm{CH}_{3}\right), 6.61(1 \mathrm{H}, \mathrm{s}, \mathrm{CH}), 7.01-7.61(8 \mathrm{H}$, $\mathrm{m}, \mathrm{ArH}) ;{ }^{13} \mathrm{C}$ NMR $\left(100 \mathrm{MHz}, \mathrm{CDCl}_{3}\right): 166.23,161.55,154.84$, $146.91,148.64,138.41,129.12,125.87,123.41,123.12$, 121.72, 121.45, 112.12, 103.11, 61.41, 56.12, 24.15, 13.42; ESI-MS: $\mathrm{m} / \mathrm{z}$ Calcd for $\mathrm{C}_{20} \mathrm{H}_{17} \mathrm{~N}_{3} \mathrm{O}_{4} \mathrm{~S} 395.46$, found $[\mathrm{M}+\mathrm{H}]^{+}$ 396.4.

2.4.3 Ethyl-2-methyl-4-(3-chlorophenyl)-4H-pyrimido[2, 1-b] [1, 3] benzothiazole-3-carboxylate (4d)

Pale yellow powder, IR: $\left(\lambda \max \mathrm{cm}^{-1}\right): 2923,1669,1563$, $1612,1221,1312,1167,983,833,755,694 \mathrm{~cm}^{-1} .{ }^{1} \mathrm{H}$ NMR $\left(400 \mathrm{MHz}, \mathrm{CDCl}_{3}\right): \delta 1.32\left(\mathrm{t}, J=7.12 \mathrm{~Hz}, 3 \mathrm{H}, \mathrm{CH}_{3}\right), 2.41(\mathrm{~s}, 3 \mathrm{H}$, $\left.\mathrm{CH}_{3}\right), 4.14\left(\mathrm{q}, J=7.12 \mathrm{~Hz}, 2 \mathrm{H}, \mathrm{OCH}_{2}\right), 6.31(\mathrm{~s}, 1 \mathrm{H}, \mathrm{CH}), 7.12$ $(\mathrm{d}, J=8.30 \mathrm{~Hz}, 1 \mathrm{H}, \mathrm{Ar}-\mathrm{H}), 7.13-7.21(\mathrm{~m}, 4 \mathrm{H}, \mathrm{Ar}-\mathrm{H}), 7.33-7.51$ $(\mathrm{m}, 3 \mathrm{H}, \mathrm{Ar}-\mathrm{H}) ;{ }^{13} \mathrm{C}$ NMR $\left(100 \mathrm{MHz}, \mathrm{CDCl}_{3}\right): \delta 15.11,22.44$, $57.21,61.11,102.41,111.23,123.11,124.18,124.78,126.56$, 127.17, 121.41, 124.81, 137.18, 133.14, 142.12, 153.79, 162.23, 167.1. ESI-MS: $\mathrm{m} / \mathrm{z} 386$. found $[\mathrm{M}+\mathrm{H}]^{+} 387.1$.

2.4.4 Ethyl-2-methyl-4-(4-dimethylamino phenyl)-4H-pyrimido[2,1-b] [1,3] benzothiazole-3-carboxylate (4e)

Pale yellow powder, IR $\left(\lambda \operatorname{max~cm} \mathrm{cm}^{-1}\right): 3023,2888,1611$, $1579,1429,1161,814-751 ;{ }^{1} \mathrm{H}$ NMR $\left(400 \mathrm{MHz}, \mathrm{CDCl}_{3}\right): \delta$ $1.32\left(3 \mathrm{H}, \mathrm{t}, J=14.11 \mathrm{~Hz}, \mathrm{CH}_{2} \mathrm{CH}_{3}\right), 2.79\left(3 \mathrm{H}, \mathrm{s}, \mathrm{CH}_{3}\right), 3.12$ $\left(6 \mathrm{H}, \mathrm{s}, \mathrm{N}\left(\mathrm{CH}_{3}\right)_{2}\right), 4.11\left(2 \mathrm{H}, \mathrm{m}, \mathrm{CH}_{2} \mathrm{CH}_{3}\right), 6.73-7.24(4 \mathrm{H}, \mathrm{m}$, $\mathrm{ArH})$, 7.72-7.926 (4H, $\mathrm{m}, \mathrm{ArH}) ;{ }^{13} \mathrm{C} \mathrm{NMR}\left(100 \mathrm{MHz}, \mathrm{CDCl}_{3}\right)$ : 167.31, 164.23, 151.91, 151.61, 134.41, 126.65, 121.23, 123.41, 122.61, 121.55, 22.11, 13.49; ESI-MS: m/z Calcd for $\mathrm{C}_{22} \mathrm{H}_{23} \mathrm{~N}_{3} \mathrm{O}_{2} \mathrm{~S} 393.54$, found $[\mathrm{M}+\mathrm{H}]^{+}$394.2.

\subsubsection{Ethyl-2-methyl-4-(4-hydroxy-3-methoxyphenyl)-4H} -pyrimido[2,1-b] [1,3] benzothiazole-3-carboxylate (4f)

Pale yellow powder, IR $\left(\lambda \max \mathrm{cm}^{-1}\right): 3023,2945,1712$, $1567,1511,1244,723 ;{ }^{1} \mathrm{H}$ NMR $\left(400 \mathrm{MHz}, \mathrm{CDCl}_{3}\right): \delta 1.33$ $\left(3 \mathrm{H}, \mathrm{t}, J=14.13 \mathrm{~Hz}, \mathrm{CH}_{2} \mathrm{CH}_{3}\right), 2.51\left(3 \mathrm{H}, \mathrm{s}, \mathrm{CH}_{3}\right), 3.6(3 \mathrm{H}, \mathrm{s}, \mathrm{Ar}-$ $\left.\mathrm{OCH}_{3}\right), 4.11-4.22\left(2 \mathrm{H}, \mathrm{m}, \mathrm{CH}_{2} \mathrm{CH}_{3}\right), 6.23(1 \mathrm{H}, \mathrm{s},-\mathrm{CH}), 6.43(1 \mathrm{H}$, $\mathrm{d}, J=5.3 \mathrm{~Hz}, \mathrm{ArH}), 6.56-6.79(2 \mathrm{H}, \mathrm{m}, \mathrm{ArH}), 7.11-7.28(4 \mathrm{H}, \mathrm{m}$, $\mathrm{ArH}), 9.56(1 \mathrm{H}, \mathrm{s}, \mathrm{OH}) ;{ }^{13} \mathrm{C} \mathrm{NMR}\left(100 \mathrm{MHz}, \mathrm{CDCl}_{3}\right): 166.12$, $161.32,152.52,146.19,145.14,136.16,131.24,121.32$, 127.34, 112.13, 113.34, 111.81, 51.25, 56.54, 23.34, 14.11 ESI-MS: $\mathrm{m} / \mathrm{z}$ Calcd for $\mathrm{C}_{21} \mathrm{H}_{20} \mathrm{~N}_{2} \mathrm{O}_{4} \mathrm{~S} 396.49$, found $[\mathrm{M}+\mathrm{H}]^{+}$ 397.2. 
2.4.6 Ethyl-2-methyl-4-(2-chlorophenyl)-4H-pyrimido[2, 1-b] [1, 3] benzothiazole-3-carboxylate $(\mathbf{4} \mathrm{g})$

Pale yellow powder, IR: $\left(\lambda \operatorname{max~cm}{ }^{-1}\right) 2922,1683,1556$, $1513,1365,1134,971,826,681 ;{ }^{1} \mathrm{H} \mathrm{NMR}\left(400 \mathrm{MHz}, \mathrm{CDCl}_{3}\right)$ : $\delta 1.22\left(\mathrm{t}, J=7.15 \mathrm{~Hz}, 3 \mathrm{H}, \mathrm{CH}_{3}\right), 2.36\left(\mathrm{~s}, 3 \mathrm{H}, \mathrm{CH}_{3}\right), 4.23(\mathrm{q}$, $\left.J=7.32 \mathrm{~Hz}, 2 \mathrm{H}, \mathrm{OCH}_{2}\right), 6.71(\mathrm{~s}, 1 \mathrm{H}, \mathrm{CH}), 7.04-7.13(\mathrm{~m}, 2 \mathrm{H}$, Ar-H), 7.11-7.23 (m, 2H, Ar-H), $7.41(\mathrm{~d}, J=7.45 \mathrm{~Hz}, 1 \mathrm{H}$, Ar-H), $7.11(\mathrm{~d}, J=8.24 \mathrm{~Hz}, 2 \mathrm{H}, \mathrm{Ar}-\mathrm{H}), 7.59(\mathrm{~d}, J=7.41 \mathrm{~Hz}$, $1 \mathrm{H}, \mathrm{Ar}-\mathrm{H}) ;{ }^{13} \mathrm{C}$ NMR $\left(100 \mathrm{MHz}, \mathrm{CDCl}_{3}\right): \delta 13.31,23.32,54.54$, 59.32, 102.87, 111.44, 121.45, 123.87, 123.33, 126.76, $128.11,129.34,129.43,130.34,131.11,138.67,139.41$, 154.21, 162.14, 163.11; ESI-MS: m/z $386[\mathrm{M}+\mathrm{H}]^{+}$.

\subsubsection{Ethyl-2-methyl-4-(4-methoxy phenyl)-4Hpyrim- ido[2,1 b] [1, 3] benzothiazole-3-carboxylate (4j)}

Pale yellow powder, IR $\left(\lambda \max \mathrm{cm}^{-1}\right): 2952,1633,1512$, $1278,1147,960-816 ;{ }^{1} \mathrm{H}$ NMR $\left(400 \mathrm{MHz}, \mathrm{CDCl}_{3}\right): \delta 1.24$ $\left(3 \mathrm{H}, \mathrm{t}, J=14.11 \mathrm{~Hz}, \mathrm{CH}_{3} \mathrm{CH}_{2}\right), 2.44\left(3 \mathrm{H}, \mathrm{s}, \mathrm{CH}_{3}\right), 3.69(3 \mathrm{H}, \mathrm{s}$, Ar- $\left.\mathrm{OCH}_{3}\right), 4.13-4.11\left(2 \mathrm{H}, \mathrm{m}, \mathrm{CH}_{3} \mathrm{CH}_{2}\right), 6.31(1 \mathrm{H}, \mathrm{s},-\mathrm{CH}), 6.67$ $(2 \mathrm{H}, \mathrm{d}, J=8.34 \mathrm{~Hz}, \mathrm{ArH}), 7.18-7.45(6 \mathrm{H}, \mathrm{m}, \mathrm{ArH}) ;{ }^{13} \mathrm{C} \mathrm{NMR}$ $\left(100 \mathrm{MHz}_{1} \mathrm{CDCl}_{3}\right): 167.45,161.34,159.34,153.54,152.11$, $138.12,133.45,128.43,126.34,121.84,122.81,123.11$, 121.65, 116.07, 113.67, 112.56, 103.67, 61.02, 56.16, 55.15, 23.68, 14.12; ESI-MS: m/z Calcd for $\mathrm{C}_{21} \mathrm{H}_{20} \mathrm{~N}_{2} \mathrm{O}_{3} \mathrm{~S} 380.47$, found $[\mathrm{M}+\mathrm{H}]^{+} 381.3$.

\subsubsection{Ethyl-2-methyl-4-(2,6-dichlorophenyl)-4Hpyrimido[2,} 1-b] [1, 3] benzothiazole-3-carboxylate $(4 \mathrm{k})$

Pale yellow powder, IR (KBr) (umax, $\mathrm{cm}^{-1}$ ): 3102, 2925, $1621,1512,1267,1113,951-814 ;{ }^{1} \mathrm{H}$ NMR $(400 \mathrm{MHz}$, $\left.\mathrm{CDCl}_{3}\right): \delta 1.02\left(3 \mathrm{H}, \mathrm{t}, J=14.16 \mathrm{~Hz}, \mathrm{CH}_{2} \mathrm{CH}_{3}\right), 2.42\left(3 \mathrm{H}, \mathrm{s}, \mathrm{CH}_{3}\right)$, $4.14\left(2 \mathrm{H}, \mathrm{m}, \mathrm{CH}_{2} \mathrm{CH}_{3}\right), 7.12-7.53(7 \mathrm{H}, \mathrm{m}, \mathrm{Ar}-\mathrm{H}) ;{ }^{13} \mathrm{C} \mathrm{NMR}$ $\left(100 \mathrm{MHz}, \mathrm{CDCl}_{3}\right): 173.84,165.45,162.78,152.02,136.34$, 132.71, 131.22. 122.42, 126.01, 125.17, 124.32, 122.23, 121.33, 117.45, 116.12, 59.11, 23.54, 14.11; ESI-MS: m/z Calcd for $\mathrm{C}_{20} \mathrm{H}_{16} \mathrm{~N}_{2} \mathrm{Cl}_{2} \mathrm{O}_{2} \mathrm{~S} 419.41$, found $[\mathrm{M}+\mathrm{H}]^{+} 419.6$.

\subsubsection{Ethyl-2-methyl-4-(2-hydroxy phenyl)-4H-pyrimido[2,1-b] $[1,3]$ benzothia- zole-3-carboxylate (4 I)}

Pale yellow powder, $\mathrm{mp} 212-215^{\circ} \mathrm{C}$; IR $\left(\lambda \max \mathrm{cm}^{-1}\right)$ : 3031 , 2814, 1672, 1569, 1479, 1242, 835-745; ${ }^{1} \mathrm{H}$ NMR $(400 \mathrm{MHz}$, $\left.\mathrm{CDCl}_{3}\right): \delta 1.34\left(3 \mathrm{H}, \mathrm{t}, J=14.17, \mathrm{CH}_{2} \mathrm{CH}_{3}\right), 2.41\left(3 \mathrm{H}, \mathrm{s}, \mathrm{CH}_{3}\right)$, 4.13-4.24 (2H, m, $\left.\mathrm{CH}_{2} \mathrm{CH}_{3}\right), 6.31(1 \mathrm{H}, \mathrm{s},-\mathrm{CH}), 6.65-7.91(8 \mathrm{H}$, $\mathrm{m}, \mathrm{ArH}), 8.8(1 \mathrm{H}, \mathrm{s}, \mathrm{OH}) ;{ }^{13} \mathrm{C} \mathrm{NMR}\left(100 \mathrm{MHz}, \mathrm{CDCl}_{3}\right): 172.87$, $166.91,163.98,156.22,152.34,134.71,125.13,127.12$, 123.91, 117.12, 59.28, 55.21, 24.13, 13.12; ESI-MS: m/z Calcd for $\mathrm{C}_{20} \mathrm{H}_{18} \mathrm{~N}_{2} \mathrm{O}_{3} \mathrm{~S} 366.34$, found $[\mathrm{M}+\mathrm{H}]^{+}$367.2.
2.4.10 Ethyl-2-methyl-4-(4-hydroxy phenyl)-4H-pyrimido $[2,1-b][1,3]$ benzothiazole-3-carboxylate $(4 n)$

Pale yellow powder, IR $\left(\lambda \max \mathrm{cm}^{-1}\right): 3291,3061,2889$, $1621,1578,1428,1381,814-756 ;{ }^{1} \mathrm{H}$ NMR $\left(400 \mathrm{MHz}, \mathrm{CDCl}_{3}\right)$ : $\delta 1.29\left(3 \mathrm{H}, \mathrm{t}, J=14.18 \mathrm{~Hz}, \mathrm{CH}_{3} \mathrm{CH}_{2}\right), 2.38\left(3 \mathrm{H}, \mathrm{s}, \mathrm{CH}_{3}\right), 2.11$ $\left(\mathrm{m}, 2 \mathrm{H}, \mathrm{CH}_{3} \mathrm{CH}_{2}\right), 6.29(1 \mathrm{H}, \mathrm{s},-\mathrm{CH}), 6.72(2 \mathrm{H}, \mathrm{d}, J=8.40 \mathrm{~Hz}$, ArH), 7.14-7.61 (5H, m, ArH), $7.62\left(1 \mathrm{H}, \mathrm{d}, J=7.69 \mathrm{~Hz}, \mathrm{ArH}_{1}\right)$, $9.32(1 \mathrm{H}, \mathrm{s}, \mathrm{OH}) ;{ }^{13} \mathrm{CNMR}\left(100 \mathrm{MHz}, \mathrm{CDCl}_{3}\right): 165.57,162.29$, $157.19,153.51,137.62,131.89,128.21,126.29,123.61$, $122.88,122.19,115.11,111.78,102.89,59.22,56.42,23.11$, 14.12; ESI-MS: $\mathrm{m} / \mathrm{z}$ Calcd for $\mathrm{C}_{20} \mathrm{H}_{18} \mathrm{~N}_{2} \mathrm{O}_{3} \mathrm{~S} 366.34$, found $[\mathrm{M}+\mathrm{H}]^{+}$367.2.

\subsubsection{Ethyl-2-methyl-4-(2,4-dichlorophenyl)-4H-pyrimido $[2,1-b][1,3]$ benzothiazole-3-carboxylate (4p)}

Pale yellow powder, IR $\left(\lambda \max \mathrm{cm}^{-1}\right): 2942,1685,1586$, $1511,1391,1242,1196,972,824,743,661 \mathrm{~cm}^{-1}$. ${ }^{1} \mathrm{H}$ NMR $\left(400 \mathrm{MHz}, \mathrm{CDCl}_{3}\right): \delta 1.26\left(\mathrm{t}, J=7.18 \mathrm{~Hz}, 3 \mathrm{H}, \mathrm{CH}_{3}\right), 2.46(\mathrm{~s}, 3 \mathrm{H}$, $\left.\mathrm{CH}_{3}\right), 4.15\left(\mathrm{q}, \mathrm{J}=7.19 \mathrm{~Hz}, 2 \mathrm{H}, \mathrm{OCH}_{2}\right), 6.72(\mathrm{~s}, 1 \mathrm{H}, \mathrm{CH}), 7.13$ (d, J=7.58 Hz, 1H, Ar-H), 7.17-7.21 (m, 1H, Ar-H), $7.26(\mathrm{~d}$, $J=7.58 \mathrm{~Hz}, 1 \mathrm{H}, \mathrm{Ar}-\mathrm{H}), 7.29-7.33(\mathrm{~m}, 1 \mathrm{H}, \mathrm{Ar}-\mathrm{H}), 7.36-7.41(\mathrm{~m}$, $2 \mathrm{H}, \mathrm{Ar}-\mathrm{H}), 7.53(\mathrm{~d}, J=9.08 \mathrm{~Hz}, 1 \mathrm{H}, \mathrm{Ar}-\mathrm{H}) ;{ }^{13} \mathrm{C} \mathrm{NMR}(100 \mathrm{MHz}$, $\mathrm{CDCl}_{3}$ ): $\delta 14.82,24.21,54.41,60.51,102.89,111.88,122.61$, $124.59,127.19,128.89,129.62,135.23,138.59,138.54$, 156.11, 163.71, 166.56. ESI-MS: m/z: $419[\mathrm{M}+\mathrm{H}]^{+}$.

\section{Results and discussion}

In the present work, our exposure was focused on the preparation of substituted $4 \mathrm{H}$-pyrimido- $[2,1-b]$ benzothiazoles (Scheme 1), which have been synthesized successfully by cyclo-condensation of ethylacetoacetate (1), aromatic aldehydes with different substituents (2) and 2-aminobenzothiazole (3) in good to excellent yields (91$96 \%)$. In the reaction, DBU was used as an organic base catalyst under micellar media which was developed by adding CPB a cationic surfactant at $60^{\circ} \mathrm{C}$ temperature. Keeping environmental concerns in mind, water was chosen as reaction solvent instead of traditional solvents.

Initially, the product was obtained in only in $15 \%$ yield in $24 \mathrm{~h}$ with DBU in SDS surfactant solution at $60^{\circ} \mathrm{C}$. After that the reactions condition of the reaction was optimized using various cationic, anionic and neutral surfactants viz. SDS, CTAC, HDBAC, TEBA, CTAB, CPB, Triton X-100 and Tween 40 (Table 1) with various organic and inorganic bases like DBU, DMAP, TEA and $\mathrm{K}_{2} \mathrm{CO}_{3}$. After optimization of surfactants and catalyst, it was concluded that, product may be obtained in excellent yield with CPB surfactant with DBU as a base in 3-4 h only. After synthesis, 


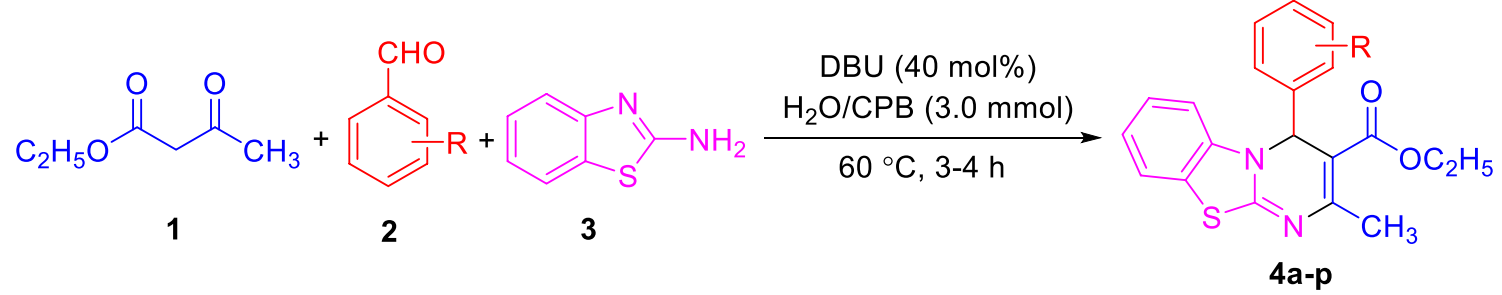

Scheme 1 Synthesis of tricyclic 4H-pyrimido[2,1-b]benzothiazoles derivatives

Table 1 Screening of surfactants

\begin{tabular}{|c|c|c|c|c|}
\hline Entry & Surfactant (mmol) & Base (mol \%) & Time (h) & Yields (\%) \\
\hline 1 & SDS (1) & - & 12 & - \\
\hline 2 & SDS (1) & DBU (10) & 12 & 15 \\
\hline 3 & $\operatorname{SDS}(2)$ & $\mathrm{DBU}(10)$ & 12 & 23 \\
\hline 4 & SDS (3) & DBU (10) & 12 & 31 \\
\hline 5 & SDS (4) & DBU (10) & 12 & 36 \\
\hline 6 & CPB (2) & DBU (10) & 12 & 41 \\
\hline 7 & CPB (2) & $\mathrm{DBU}(20)$ & 12 & 53 \\
\hline 8 & CPB (2) & $\mathrm{DBU}(30)$ & 6 & 69 \\
\hline 9 & CPB (3) & $\mathrm{DBU}(30)$ & 4 & 84 \\
\hline 10 & CPB (3) & DBU (40) & 3 & 93 \\
\hline 11 & CPB (4) & $\mathrm{DBU}(40)$ & 3 & 76 \\
\hline 12 & CPB (3) & $\mathrm{DBU}(50)$ & 3 & 51 \\
\hline 13 & CPB (3) & $\mathrm{K}_{2} \mathrm{CO}_{3}(40)$ & 6 & - \\
\hline 14 & CPB (3) & DMAP (40) & 3 & 12 \\
\hline 15 & CPB (3) & TEA (40) & 3 & 17 \\
\hline 16 & CTAB (3) & $\mathrm{DBU}(40)$ & 3 & 54 \\
\hline 17 & HDBAC (3) & $\mathrm{DBU}(40)$ & 3 & 65 \\
\hline 18 & TEBA (3) & $\mathrm{DBU}(40)$ & 3 & 50 \\
\hline 19 & CTAC (3) & $\mathrm{DBU}(40)$ & 3 & 47 \\
\hline 20 & Triton X-100 (60) & DBU (40) & 12 & - \\
\hline 21 & Tween 40 (60) & DBU (40) & 12 & - \\
\hline
\end{tabular}

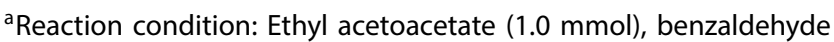
$(1.0 \mathrm{mmol}), 2$-aminobenzothiazole $(1.0 \mathrm{mmol})$ under DBU catalysis in micellar media at $60^{\circ} \mathrm{C}$ for $3-12 \mathrm{~h}$

the products were recrystallized using hot ethanol and characterized by ${ }^{1} \mathrm{H}-\mathrm{NMR}$,

${ }^{13} \mathrm{C}-\mathrm{NMR}$, IR and Mass spectral studies which were in close agreement with the product which was synthesized by a method reported in literature. After optimization of reaction conditions for the reaction, sixteen derivatives with various substitution have designed and synthesized in good to excellent yields under standard reaction conditions and concluded that, from the variations of the yields of the products (4a-p), the presence of an electron-donating group in the substituted aldehydes used facilitates the reaction, and lower yield of the product is obtained when electron-withdrawing group is used. Within the perspective, the similar results were obtained when gram scale experiment was performed for the same reaction. The physico-chemical data of all the synthesized products have been illustrated in the Table 2 .

In order to check the greenness of the reaction, various credentials for green chemistry of the newly developed protocol have been calculated using the well defined formulas reported in literature [42-44].

The calculated green credential indices contain atom economy (AE), atom efficiency (AEf), carbon efficiency $(C E)$, reaction mass efficiency (RME), optimum efficiency (OE) and E-factor. Atom economy (90.68-92.07) and atom efficiency (83.40-87.98) for the method measures are obtained. The process shows a good profile of calculated carbon efficiency (100\%). Reaction mass efficiency (83.4788.37) which may be regarded as the most useful metric to determine the greenness of the developed process; calculations of RME also support excellent green credential of the present method. Similarly, the calculated E-factors $(\mathrm{g} / \mathrm{g})$ obtained in the range from $0.13-0.20$, which convincingly indicate considerable green aspects of the present procedure.

\subsection{Gram-Scaled up reaction}

In the perspective, for checking the practicality of the reaction, a gram scaled up experiment was performed at $5.0 \mathrm{mmol}$ scale (Scheme 2) under optimized reaction conditions, the results of the reaction are quite similar to the small scale reaction in $4 \mathrm{~h}$.

\subsection{Competitive experiment}

By encouraging the results obtained, a competitive experiment was performed (Scheme 3) for investigation of the variation of the yields of the products, which alters due to starting materials.

In the experiment, ethyl acetoacetate $\mathbf{1}$ and 2-aminobenzothiazole $\mathbf{3}$ were permitted to react with an equimolar mixture of $p$-nitrobenzaldehyde and $p$-methoxybenzaldehyde $\mathbf{2}$, products $\mathbf{4 b}$ and $\mathbf{4} \mathbf{h}$ were obtained in $18 \%$ and $67 \%$ yields respectively, which was concluded that 


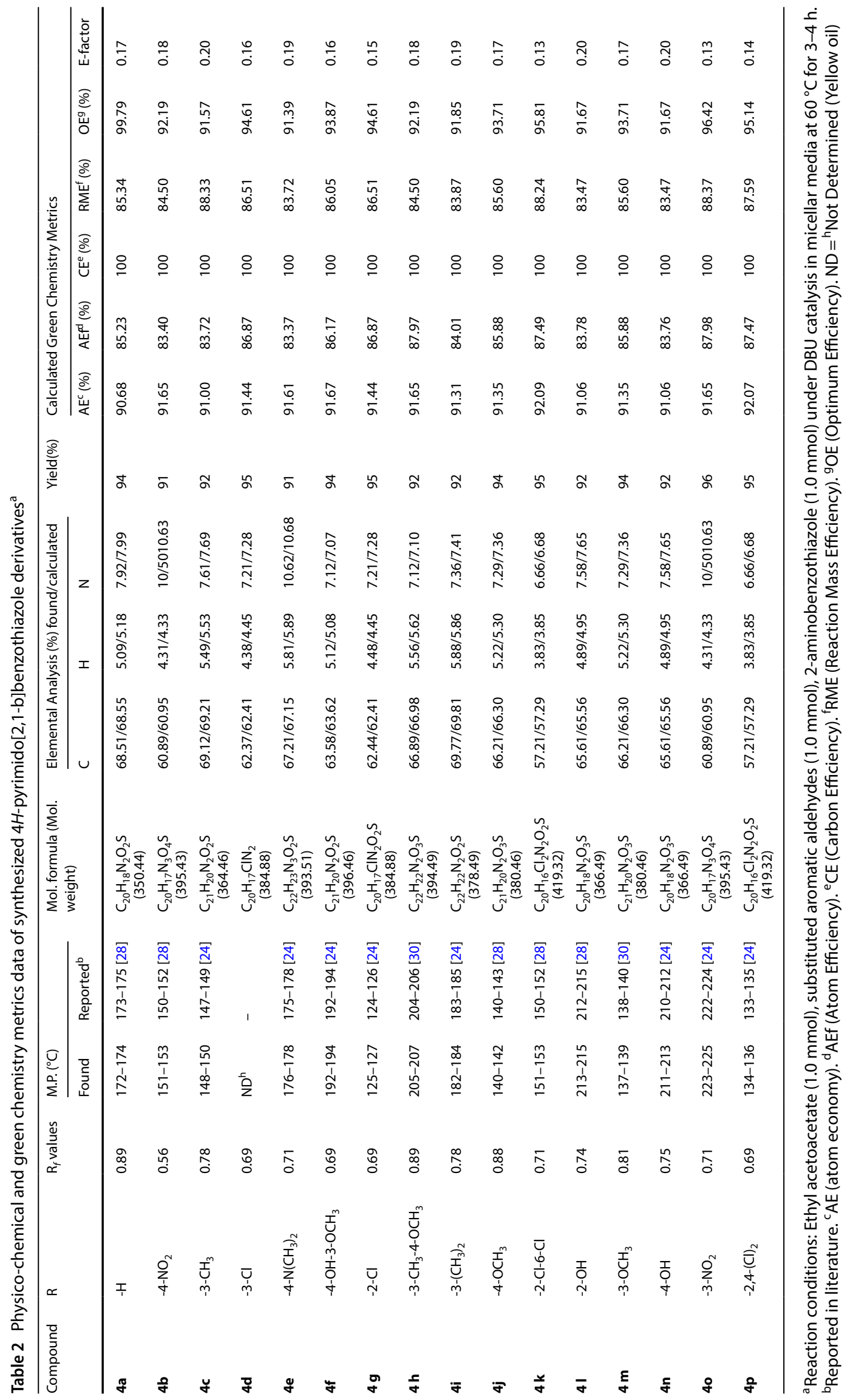


when the substrates with electron-donating and electronwithdrawing groups were permitted to react individually, they give better yields as compared to a substrate with electron-withdrawing group. Nonetheless, in the mixture, substrate with electron-donating groups not only reacts faster, but also gives higher yields of the product as compared to substrates with electron-withdrawing group. proceed via formation of carbocations, which is easily formed by DBU. The lone pair of electron present of nitrogen in 2-aminobenzothiazole may attack on carbocations as a result of which an intermediate is formed, which further reacts with ethyl acetoacetate followed by cyclization gives targeted molecule.

\subsection{Mechanistic study}

For the reaction, a plausible mechanistic pathway is depicted in Fig. 3 using DBU in CPB surfactants may

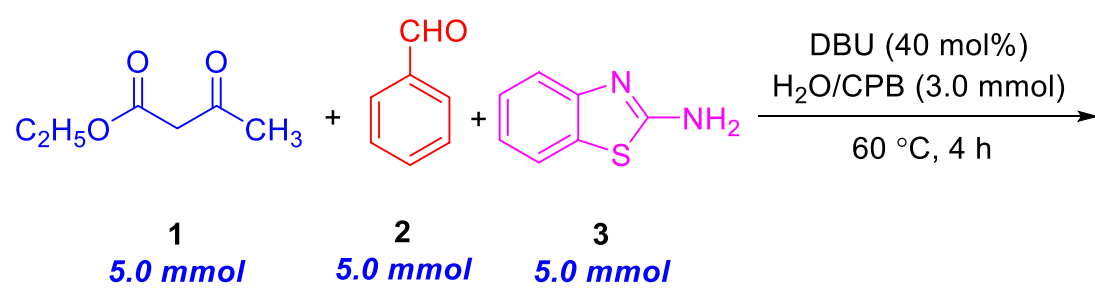<smiles>CCOC(=O)C1=C(C)N=C2Sc3ccccc3N2C1c1ccccc1</smiles>

$4 a$

$89 \%$ yield

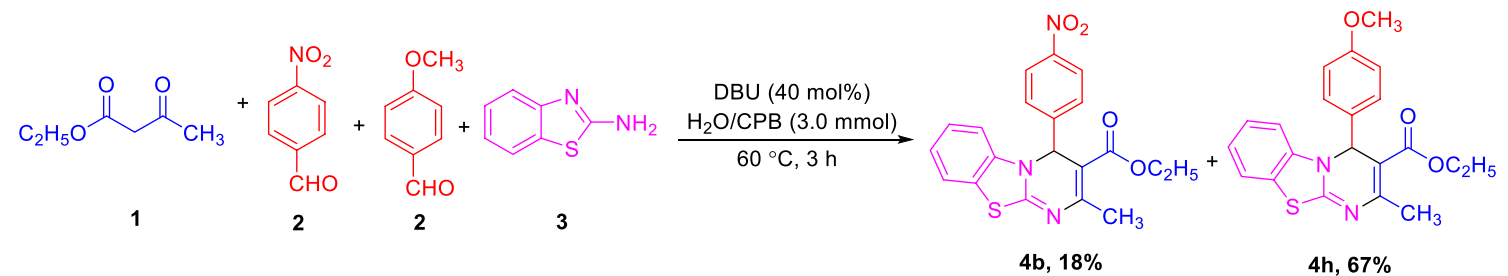

Scheme 3 Competitive experiment for tricyclic $4 H$-pyrimido[2,1-b]benzothiazole synthesis

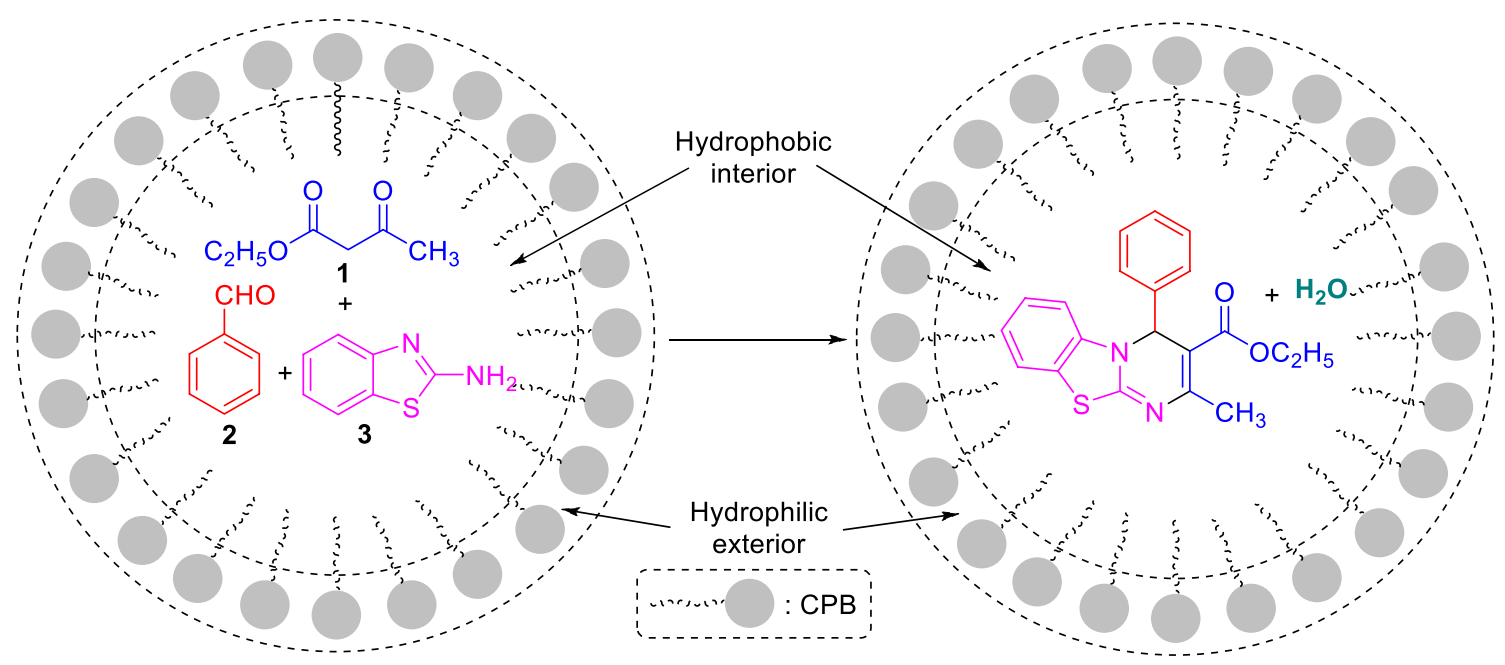

Fig. 3 Mechanistic picture diagram representing the role of $\mathrm{CPB}$ 


\section{Conclusions}

In conclusion, we have established a multicomponent three component and green synthesis of biologically active molecule triheterocyclic $4 H$-pyrimido $[2,1-b]$ benzothiazole and its derivatives by the cyclocondensation reaction of $\beta$-ketoester, aromatic aldehydes and 2 -aminobenzothiazole as reagents in aqueous miceller medium, using CPB as the surfactant and DBU as a organic base. The notable advantages of this present protocol include use of easily available cheap starting materials and ecofriendly greener catalyst, metal-free one-pot synthesis, energy-efficiency, short reaction durations, clean reaction profiles, operational simplicity, ease of product purification without column chromatography, large-scale synthetic applicability, good to excellent yields, high atom-economy and low E-factor over reported protocols which provides a useful platform to synthesize the novel biologically active scaffolds in molecular designing.

Acknowledgements Authors are thankful to Dr. K.N. Modi University, Newai, Rajasthan, India for providing seed grant No. DKNMU/R\&D/2019/01.

\section{Compliance with ethical standards}

Conflict of interest Authors have no conflict of interest.

\section{References}

1. Andrade CKZ, Alves LM (2005) Environmentally benign solvents in organic synthesis: current topics. Curr Org Chem 9:195-218

2. Chanda A, Fokin VV (2009) Organic synthesis "On water". Chem Rev 109:725-748

3. Chao-Jun L, Liang C (2006) Organic chemistry in water. Chem Soc Rev 35:68-82

4. Lindstrom UM (2002) Stereoselective organic reactions in water. Chem Rev 102:2751-2772

5. Dömling A, Wang W, Wang K (2012) Chemistry and biology of multicomponent reactions. Chem Rev 112:3083-3135

6. Jiang B, Rajale T, Wever W, Tu S-J, Li G (2010) Multicomponent reactions for the synthesis of heterocycles. Chem-Asian J 5:2318-2335

7. Ramon DJ, Yus M (2005) Asymmetric multicomponent reactions (AMCRs): the new frontier. Angew Chem Intl Ed 44:1602-1634

8. Tuch A, Wallé $S$ (2002) Multicomponent reactions. In: Nicolaou KC, Hanko R, Hartwig W (eds) Handbook of combinatorial chemistry, vol 2. Wiley-VCH, Weinheim, pp 685-705

9. Chen J, Liu W, Ma J, Xu H, Wu J, Tang X, Fan Z, Wang P (2012) Synthesis and properties of fluorescence dyes: tetracyclic pyrazolo[3,4-b]pyridine-based coumarin chromophores with intramolecular charge transfer character. J Org Chem 77:3475-3482

10. Borah P, Bhuyan PJ (2013) Synthesis of some novel spiro substituted pyrido[2,3-c] coumarins by exploring 'tertiary amino effect' reaction strategy. Tetrahedron Lett 54:6949-6951
11. Khan IA, Kulkarni MV, Gopal M, Shahabuddin MS, Sun CM (2005) Synthesis and biological evaluation of novel angularly fused polycyclic coumarins. Bioorg Med Chem Lett 15:3584-3587

12. Pal S, Khan MN, Karamthulla S, Choudhury LH (2013) Molecular iodine catalyzed one-pot multicomponent reactions for the synthesis of dihydrochromeno[4,3-b]pyrazolo[4,3-e]pyridin-6(7H)ones. RSC Adv 3:15705-15711

13. Kutyrev A, Kappe T (1999) Methanetricarboxylates as key reagents for the simple preparation of heteroarylcarboxamides with potential biological activity. Part 2[1]. Reaction of methane-tricarboxylates with 2-aminopyridine, 2-aminopyrimidine, 2-amino-thiazole and 2-aminobenzothiazole. J Heterocyclic Chem 36(1):237-240

14. Gineinah MMM (2001) 6,7 and 8-(5-Aryl-1-phenyl-2-pyrazolin3-yl) imidazo and pyrimido $[2,1-b]$ benzothiazoles as novel anticonvulsant agents. Sci Pharma 69(1):53-61

15. El-Sherbeny MA (2000) Synthesis of certain pyrimido[2,1-b] benzothiazole and benzothiazole[2,3-b]quinazoline derivatives for in vitro antitumor and antiviral activities. ArzneimittelForschung Drug Res 50(9):848-853

16. Tanabe Y, Kawai A, Yoshid Y, Ogure M, Okumura H (1997) Preparation of fused thiadiazolo- and imidazobenzothiazoles from 2-aminobenzothiazoles. Their fungicidal activity. Heterocycles 45(8):1579-1588

17. Wade JJ, Tose CB, Matson CJ, Stelzer CL (1983) Synthesis and antiallergic activity of some acidic derivatives of 4H-Pyrimido[2,1-b]benzazol-4-ones. J Med Chem 26(4):608-611

18. Agarwal S, Agarwal DK, Gandhi D, Goyal K, Goyal P (2018) Multicomponent one-pot synthesis of substituted $4 H$-pyrimido[2,1-b] $[1,3]$ benzothiazole curcumin derivatives and their antimicrobial evaluation. Lett Org Chem 15(10):863-869

19. Bhoi MN, Borad MA, Jethava DJ, Acharya PT, Pithawala EA, Patel CN, Pandya HA, Patel HD (2019) Synthesis, biological evaluation and computational study of novel isoniazid containing 4H-Pyrimido[2,1-b]benzothiazoles derivatives. Eur J Med Chem 177:12-31

20. Deshmukh VK, Yadav MR, Chaudhari SR (2013) Synthesis and anti-inflammatory activity of substituted pyrimido[2,1-b][1,3] benzothiazole derivatives. JPR BioMedRx Intl J 1(6):574-578

21. Caleta I, Grdisa M, Mrvos-Sermek D, Cetina M, Tralic-Kulenovic V, Pavelic K, Karminski-Zamola G (2004) Synthesis, crystal structure and antiproliferative evaluation of some new substituted benzothiazoles and styrylbenzothiazoles. IL Farmaco 59:297-305

22. Hassan AY (2009) Some reactions of 2-cyanomethyl-1,3-benzothiazole with expected biological activity. Phos Sulfur Silicon Relat Elem 184:2856-2869

23. Borthwick AD, Davies DE, Ertl FF, Exall AM, Haley TM, Hart GJ, Jackson DL, Parry NR, Patikis A, Trivedi N, Weingarten GG, Woolven JM (2003) Design and synthesis of pyrrolidine-5,5'-translactams (5-oxo-hexahydropyrrolo[3,2-b]pyrroles) as novel mechanism based inhibitors of human cytomegalovirus protease. 4 . antiviral activity and plasma stability. J Med Chem 46:4428-4449

24. Safajoo N, Mirjalili BBF, Bamoniri A (2019) $\mathrm{Fe}_{3} \mathrm{O}_{4} @$ nano-cellulose/ $\mathrm{Cu}$ (II): a bio-based and magnetically recoverable nano-catalyst for the synthesis of $4 \mathrm{H}$-pyrimido[2,1-b] benzothiazole derivatives. RSC Adv 9:1278-1283

25. Suresh AJ, Bharathi K, Surya PR (2018) Design, synthesis, characterization and biological evaluation of some novel benzothiazole derivatives as anti tubercular agents targeting glutamine synthetase-I. J Pharm Chem Bio Sci 5(4):312-319

26. Nagarapu L, Gaikwad HK, Palem JD, Venkatesh R, Bantu R, Sridhar B (2013) Convenient approach for the one-pot, three-component synthesis of triheterocyclic $4 \mathrm{H}$-pyrimido[2,1-b] benzothiazole derivatives using TBAHS. Synthetic Commun 43:93-104

27. Atar $A B$, Jeong YS, Jeong YT (2014) Iron fluoride: the most efficient catalyst for one-pot synthesis of $4 \mathrm{H}$-pyrimido[2,1-b] 
benzothiazoles under solvent-free conditions. Tetrahedron 70:5207-5213

28. Shaabani A, Rahmati A, Naderi S (2005) A novel one-pot three-component reaction: synthesis of triheterocyclic $4 \mathrm{H}$-pyrimido[2,1-b]benzazoles ring systems. Bioorg Med Chem Lett 15:5553-5557

29. Heravi MM, Hashemi E, Beheshtiha YS, Kamjou K, Toolabi M, Hosseintash N (2014) Solvent-free multicomponent reactions using the novel $\mathrm{N}$-sulfonic acid modified poly(styrene-maleic anhydride) as a solid acid catalyst. J Mol Catal A: Chem 392:173-202

30. Azad S, Mirjalili BBF (2016) $\mathrm{Fe}_{3} \mathrm{O}_{4} @$ nano-cellulose/TiCl: a biobased and magnetically recoverable nano-catalyst for the synthesis of pyrimido[2,1-b]benzothiazole derivatives. RSC Adv 6:96928-96934

31. Dake SA, Tekale SU, Sarda SR, Jadhav WN, Bhusare SR, Pawar RP (2008) Anhydrous zinc chloride: an efficient catalyst for one pot synthesis of 2,3,4,12-tetrahydro-benzo-[4,5]-thiazolo-[2,3b]quinazolin-1-ones. ARKIVOC 17:241-247

32. Rao DGB, Acharya BN, Verma SK, Kaushik MP (2011) N, $\mathrm{N}^{\prime}$-Dichlorobis(2,4,6-trichlorophenyl)urea (CC-2) as a new reagent for the synthesis of pyrimidone and pyrimidine derivatives via Biginelli reaction. Tetrahedron Lett 52:809-812

33. Meshram HM, Kumar AS, Kumar GS, Swetha A, Reddy BC, Ramesh P (2012) Boric acid promoted an efficient and practical synthesis of fused pyrimidines in aqueous media. Der Pharma Chemica 4:956-960

34. Dai C, Xie Z, Qing X, Luo N, Wang C (2020) DBU-mediated annulation of 2-aryl-3-nitro- $2 \mathrm{H}$-chromenes with 1,3-cyclohexanediones for the synthesis of benzofuro[2,3-c] chromenone derivatives. Mol Diver 24(1):191-200

35. Sorella GL, Strukul G, Scarso A (2015) Recent advances in catalysis in micellar media. Green Chem 17:644-683

36. Lal J, Singh S, Rani P (2020) Synthesis of 2-aminobenzothiazolomethyl naphthols using L-valine organocatalyst: an efficient, versatile and biodegradable catalyst. Chem Africa 3(1):53-60
37. Lal J, Gupta SK, Thavaselvam D, Agarwal DD (2016) Synthesis and pharmacological activity evaluation of curcumin derivatives synthesis and pharmacological activity evaluation of curcumin derivatives. Chin Chem Lett 27:1067-1072

38. Lal J, Gupta SK, Thavaselvam D, Agarwal DD (2012) Design, synthesis, synergistic antimicrobial activity and cytotoxicity of 4-aryl substituted 3,4-dihydropyrimidinones of curcumin. Bioorg Med Chem Lett 22:2872-2878

39. Lal J, Gupta SK, Agarwal DD (2012) Chitosan: an efficient biodegradable and recyclable green catalyst for one-pot synthesis of 3,4-dihydropyrimidinones of curcumin in aqueous media. Catal Commun 27:38-43

40. Lal J (2015) Sphalerite: a natural catalyst for an efficient synthesis of 2-aminobenzothiazolomethyl naphthol derivatives under solvent-free conditions. Intl J Inter Res 2(1):5-9

41. Balyan S, Sharma R, Lal J (2020) Microwave-assisted, efficient and eco-friendly synthesis of novel $3 \mathrm{H}$-benzo[b][1,4]diazepine derivatives using basic alumina as a reusable catalyst. Chem Africa 3(1):35-44

42. Sheldon RA (2018) Metrics of green chemistry and sustainability: past, present and future. ACS Sustain Chem Engg 6:32-48

43. Abou-Shehada S, Mampuys P, Maes BUW, Clark JH, Summerton $L$ (2017) An evaluation of credentials of a multicomponent reaction for the synthesis of isothioureas through the use of a holistic green metrics toolkit. Green Chem 19:249-258

44. Willis NJ, Fisher CA, Alder CM, Harsanyi A, Shukla L, Adams JP, Sandford G (2016) Sustainable synthesis of enantiopure fluorolactam derivatives by a selective direct fluorination-amidase strategy. Green Chem 18:1313-1318

Publisher's Note Springer Nature remains neutral with regard to jurisdictional claims in published maps and institutional affiliations. 